\title{
Pathogenic measles viruses cannot evolve to bypass vaccine-induced neutralizing antibodies
}

Miguel Ángel Muñoz-Alía ${ }^{1 *}$, Rebecca A. Nace ${ }^{1}$, Lianwen Zhang ${ }^{1}$, Stephen J. Russell ${ }^{1,2}$.

${ }^{1}$ Department of Molecular Medicine, Mayo Clinic, Rochester, MN, USA

${ }^{2}$ Department of Molecular Medicine and Division of Hematology, Mayo Clinic, Rochester, MN, USA

* Correspondence to alia.miguel@ mayo.edu (MAM-A); sjr@ mayo.edu (SJR) 


\section{$1 \underline{\text { Abstract }}$}

2 After centuries of pestilence and decades of global vaccination, measles serotypes capable of

3 evading vaccine-induced immunity have not emerged. Here, by systematically building

4 mutations into the H-glycoprotein of an attenuated measles strain and assaying for serum

5 neutralization, we show that virus evolution is severely constrained by the existence of numerous

6 codominant H-glycoprotein antigenic sites, some critical for binding to pathogenicity receptors

7 SLAMF1 and Nectin-4. We further demonstrate the existence in serum of protective

8 neutralizing antibodies targeting codominant F-glycoprotein epitopes. Calculations suggest that

9 evolution of pathogenic measles viruses capable of escaping serum neutralization in vaccinated

10 individuals is a near-zero probability scenario. 
11 Measles $(\mathrm{MeV})$ is a highly transmissible airborne pathogen that spreads systemically and causes

12 transient immune suppression $(1,2)$. Childhood infection is associated with significant mortality

13 and MeV elimination remains a high priority for the World Health Organization (3). MeV cell

14 entry via the immune cell receptor SLAMF1 (CD150) drives MeV immunopathogenesis (4),

15 whereas a second epithelial cell receptor, NECTIN-4 (PVRL4) is exploited for virus

16 transmission (5). Receptor attachment and virus entry are mediated by the concerted action of the

17 hemagglutinin (H) and fusion (F) surface glycoproteins (2). The MeV polymerase has a high

18 mutation rate and a correspondingly high frequency of monoclonal nAb-escape mutants but has

19 nevertheless remained monotypic $(6,7)$. Vaccination with a lab-adapted isolate of the genotype

20 A strain $\mathrm{MeV}$, isolated from the throat of David Edmonston in 1954, still confers full protection

21 against all currently circulating genotypes. The evolutionary stability of MeV remains poorly

22 understood but could be due to the inability of its surface glycoproteins to tolerate sequence

23 modification (8-10), the multiplicity of B cell epitopes displayed on its surface and/or the low

24 likelihood of mutational escape from combined B and T cell mediated antiviral defences.

26 Here, to elucidate the serotypic constraints on $\mathrm{MeV}$ evolution, we introduced mutated

27 hemagglutinin glycoproteins into the Moraten $\mathrm{MeV}$ vaccine whose attenuated phenotype is

28 highly stable with no recorded cases of reversion to pathogenicity or person to person

29 transmission (11). Mechanistically, vaccine attenuation is multifactorial, arising from the

30 acquisition of CD46 tropism (12), inactivating mutations in $\mathrm{V}$ and $\mathrm{C}$ immune combat proteins,

31 mutations in the $\mathrm{L}$ (polymerase) protein (13), and mutations in noncoding sequences (14). 
33 To facilitate the detailed analysis of MeV-H neutralization we assembled a comprehensive set of

3430 published anti-MeV-H monoclonal antibodies known to neutralize virus infectivity. For those

35 antibodies with unknown target epitopes we propagated the virus in the presence of the antibody,

36 selected escape mutants and sequenced them for epitope identification. Subsequently, we

37 introduced increasing numbers and varying combinations of epitope escape mutations into an

38 antigenically advanced and relatively malleable $\mathrm{MeV}-\mathrm{H}$ protein obtained from the $\mathrm{H} 1$ genotype

39 of $\mathrm{MeV}$ (15) which, to facilitate the evaluation of mutants with disrupted SLAMF1 and

40 NECTIN-4 receptor binding sites, had been engineered to bind to CD46 by introducing

41 mutations N481Y, H495R, and S546G (Fig. S1). We thereby generated a CD46-tropic MeV

42 (MeV-H $\Delta 8)$ in which all 7 previously reported antigenic sites (15), plus an $8^{\text {th }}$ site (IIc)

43 identified in the course of the current study were successfully disrupted (Fig. S2 and Fig. S3).

44 Neutralization assays confirmed that viruses encoding the MeV-H $\Delta 8$ showed a decreased

45 susceptibility to neutralization by all 30 mAbs (Fig. 1A and Fig. S4).

47 Interim analysis of the neutralization of mutated viruses by anti-MeV $\mathrm{H}$ antisera had revealed

48 that disruption of 4 or fewer antigenic sites did not abrogate polyclonal antibody neutralization

49 (15-17). However, in MeV-H $\Delta 8$ we observed an 8-fold reduction in the neutralization titer of

50 mouse anti-H antiserum (Fig. 1B). We therefore back-mutated the disrupted antigenic sites to

51 create a panel of $\Delta 7$ viruses, each one uniquely presenting a single intact antigenic site.

52 Interestingly, with the exception of the $\Delta 7$ viruses retaining antigenic sites $\Phi$, IIa or IIb, all the

53 other four $\Delta 7$ viruses were serologically indistinguishable from the parental virus, (Fig. 1B).

54 These results were replicated using sera from MeV-H-immunized rabbits (Fig. S5). Thus, 
55 simultaneous disruption of at least five antigenic sites is required to manifest resistance to

56 neutralization by MeV-H antisera.

57

58 We next sought to determine whether polyclonal sera from measles vaccinees paralleled the

59 above reactivity of mouse and rabbit antisera. Serum samples from Dutch individuals were tested

60 before and after depletion of MeV-F reactive antibodies (Fig. S6). Whereas MeV-F-depleted

61 human serum retained its neutralization potency against viruses displaying $\mathrm{MeV}-\mathrm{H} \mathrm{A}$, the $\Delta 8$

62 viruses were less readily neutralized (7-fold reduced susceptibility after averaging individual

63 titers) (Figure 1C).

64

65 Since antigenic site III is known to overlap with the SLAMF1 and NECTIN4 receptor-

66 interacting surfaces of the MeV-H (18), the elimination of B cell epitopes in this region was

67 expected to negatively impact binding to these critical pathogenicity determining receptors. We

68 therefore examined the receptor tropisms of the $\Delta 8$ variant and of the $\Delta 7$ variants exhibiting

69 reduced susceptibility to serum neutralization (Fig. S7). None of these viruses was able to

70 mediate infection via SLAMF1, indicating that the emergence of a pathogenic measles strain

71 with reduced susceptibility to serum neutralization would require the virus to reconfigure the

72 receptor interacting residues in antigenic site III in such a way as to destroy the dominant B cell

73 epitopes it shares with existing MV vaccine strains, while retaining NECTIN4 and SLAMF1

74 receptor binding affinities.

75

76 Having demonstrated that the multiply mutated $\mathrm{MeV}-\mathrm{H} \Delta 8$ protein could not interact with

77 pathogenicity-determining $\mathrm{MeV}$ receptors, we next sought to eliminate the confounding effect of 
neutralizing antibodies recognizing the MeV-F glycoprotein (Fig. 2). To achieve this, we

79 substituted the MeV-F gene with the corresponding gene from canine distemper virus (CDV). Mutations were introduced into the CDV-F and MeV-H coding sequences to restore and

81 optimize the fusogenicity of this heterologous F/H pairing (Fig. S8). This virus, hereafter termed

82 MR (Moraten Resurfaced) was subjected to Sanger sequencing and protein composition analysis

83 to confirm its identity (Fig. S9). Propagation of the MeV MR virus on Vero cells was

84 significantly slowed versus the comparable virus incorporating an unmodified MeV-H genotype

85 A protein (Fig. S10A). Propagation of MeV-H $\Delta 8$ with the parental MeV-F glycoprotein was

86 similarly impaired, indicating that mutation of multiple surface residues in $\mathrm{MeV}-\mathrm{H}$ compromised

87 protein folding and/or function (Fig. S10B).

89 We next tested whether MeV MR was resistant to human serum from vaccinated Dutch ( $\mathrm{n}=13$,

90 cohort 1), Minnesotan ( $\mathrm{n}=6$, cohort 2$)$ and Hispanic individuals ( $\mathrm{n}=4$, cohort 3 ) using an

91 improved luciferase-based infection neutralization assay (Fig. S11 and Fig. 2A). ND50 values of

92 the tested serum samples gave an overall geometric mean neutralization titer $4 \log _{2}$-fold lower

$93 \mathrm{MeV}-\mathrm{MR}$ versus MeV-A (Fig. 2B), suggesting that resistance to neutralization of the MeV MR

94 is fully manifest only at or below a MeV-A ND 50 titer of $1591 \mathrm{mIU} / \mathrm{mL}$ (Fig. 2C).

96 Interestingly, measles-immune serum does retain some level of neutralizing activity against the

97 MR virus, suggesting that it may also contain protective antibodies directed against subdominant

98 epitopes in the $\mathrm{H}$ glycoprotein. To test this, we inoculated the MR or A viruses into

99 immunocompetent HuCD46Ge-IFNar ${ }^{\mathrm{KO}}$ mice, harvested sera 4 weeks later and tested for the

100 presence of $\mathrm{IgG}$ antibodies directed against the nucleocapsid (MeV-N) or against the MeV-H 
101 proteins of pathogenic MeVs (Fig. S12). Interestingly, the data confirm that antisera raised

102 against MeV MR do weakly crossreact with wild type MeV-H, indicating that subdominant B

103 cell epitopes may play a significant role in $\mathrm{MeV}$ defense. Conversely, antibodies raised against

104 the A virus were able to crossreact weakly with subdominant epitopes in the MeV-H $\Delta 8$ protein.

105

106 Since MeV-MR is partially resistant to neutralization by measles-immune human sera, it was

107 important to confirm that, like $\mathrm{MeV}-\Delta 8$, it lacks the ability to use the pathogenicity-determining

108 receptors SLAMF1 and NECTIN4, and enters cells exclusively via CD46. This was confirmed

109 using CHO cells expressing CD46, NECTIN4, or SLAMF1 where, unlike MeV-A, MeV-MR

110 infected only cells expressing CD46 (Fig. 4A and Fig. S13). This selective tropism is

111 particularly interesting because previous reports claimed that NECTIN4 tropism could not be

112 eliminated independently of CD46 tropism $(19,20)$ (Fig. S13). We therefore measured the

113 densities of CD46 and NECTIN4 receptors on our respective CHO cell transfectants and found

114 them to be equivalent (Fig. S1B). Cotransfecting plasmids encoding MeV-F and MeV-H $\Delta 8$

115 confirmed that intercellular fusion occurred only in CD46-positive and not NECTIN4-positive

116 CHO cells (Fig. 4B) and was similar with CD46 of nonhuman primate origin (Fig. S15).

118 Further mechanistic studies into the discrimination of CD46 over NECTIN-4 showed that MeV-

$119 \mathrm{H} \triangle 8$ bound more strongly to CD46 than to NECTIN4, and negligibly to SLAMF1. This

120 contrasted with the binding pattern for MeV-H A (Fig. 4C) and suggested that MeV-H $\Delta 8$

121 discriminates between CD46 and NECTIN4 via differences in its binding affinities to each of

122 these receptors. We identified no second-site mutations in known contact residues to explain this

123 unexpected segregation of CD46 and NECTIN4 tropisms and therefore postulate that the 
124 phenotype may be partially attributable to specific noncontact residues in the $\mathrm{MeV}-\mathrm{H}$ protein of 125 genotype $\mathrm{H} 1$.

126 Measles-immune human serum is known to negate seroconversion in infants during the first year

127 of life and negates the therapeutic effect of systemically administered oncolytic MeV. Hence,

128 we sought to investigate the impact of passive immunization on the infectivity of the MR virus

129 versus the $\mathrm{MeV}$ vaccine. Whereas passive immunization with $\mathrm{MeV}$ antisera led to a decrease in

130 luciferase signal from MeV A, there was no reduction in the case of the MeV MR (Fig. S16).

131 Accordingly, systemically administered MeV MR in tumor-bearing mice could reach its target

132 (the tumor cells), in the presence of passive antibodies (Fig. S17).

134 In summary, by engineering the surface glycoproteins of a MeV vaccine, we have elucidated a 135 number of critical factors determining the remarkable antigenic stability of this monotypic virus.

136 First, $\mathrm{MeV}$ has numerous immunologically codominant antigenic sites on its $\mathrm{H}$ and $\mathrm{F}$ surface

137 glycoproteins. Second, antibodies to each of the seven known antigenic regions on the $\mathrm{H}$

138 glycoprotein are capable of neutralizing virus infectivity. Third, MeVs retaining even a single

139 immunodominant antigenic site on the $\mathrm{H}$ glycoprotein remain fully susceptible to neutralization

140 by measles immune human serum. And fourth, the receptor binding surface of the MeV-H

141 glycoprotein is itself an immunodominant antigenic site. Hence MeVs cannot escape their

142 susceptibility to neutralization by measles-immune human serum unless they also lose their

143 tropism for the pathogenicity-determining receptors SLAMF1 and NECTIN4.

145 Given that a minimum of 5 immunodominant antigenic sites must be disrupted to impact the

146 susceptibility of MeV-H to serum neutralization and that the error rate of the MeV polymerase is 
147 approximately $1 \times 10^{-5}$ mutations per site per round of genome replication (21), we estimate the

148 probability of such a virus arising to be of the order of 1 in $10^{25}$ progeny virions (weighing

149 approximately 10,000,000 kilograms)(22). Further, the simultaneous disruption of fewer than

150 five major antigenic sites would confer no selective advantage on the virus, making stepwise

151 evolution an unrealistic pathway to achieve a neutralization-resistant phenotype. However, even

152 a virus insensitive to anti-H antibodies would still be efficiently neutralized by MeV-F-specific

153 antibodies in immune human sera and, even more critical, would lack the SLAMF1 and

154 NECTIN4 receptor tropisms required for pathogenicity and transmission. We therefore conclude

155 there is a near-zero probability for the accidental emergence of a pathogenic MeV capable of

156 evading vaccine-induced immunity. 
Acknowledgments: We sincerely thank the following individuals: Mark J. Federspiel, PhD, for mAb c148. Ianko D. Iankov, MD, $\mathrm{PhD}$, for mAb 20H6 and Professor Claude Muller, $\mathrm{MD}$, for the remaining murine $\mathrm{nAb}$ as well as discussions. Roberto Cattaneo, $\mathrm{PhD}$, for Vero/dogSLAM, measles virus antigenome plasmids and rabbit anti-MeV antibodies for Western blotting. Rik L. de Swart, PhD, for the Mel-JuSo cell lines, human serum samples and antibodies C28-10-8 and F3-5. Mayo Clinic Biobank, for serum samples from the Minnesotan cohort. M. Cristine Charlesworth, PhD, and Benjamin J. Madden (Mayo Clinic Proteomics Core) for purification

164 and mass spectrometry of soluble receptors-Fc. Zene Matzuda, MD, PhD, DSc, for the split 165 luciferase plasmids. Eugene Bah, for his assistance with the initial quantitative fusion assays and useful discussion. We also thank the Mayo Clinic Biosafety Committee for their critical reading

167 of the manuscript and helpful discussion. Funding: This work was funded by grants from $\mathrm{Al}$ and 168 Mary Agnes McQuinn and Mayo Clinic. The funders had no role in study design, data collection 169 and interpretation, or the decision to submit the work for publication. Author contributions:

170 Conceptualization: M.A.M.-A., S.J.R.; Methodology: M.A.M.-A., S.J.R.; Validation: M.A.M.-A.

171 S.J.R; Investigation: M.A.M.-A., and LZ.; Analysis: M.A.M.-A., S.J.R.; Resources: M.A.M.-A.,

172 S.J.R.; Writing, review and editing: M.A.M.-A., S.J.R. Funding acquisition: S.J.R. Approval of

173 the final manuscript: All authors. Competing interests: MAM-A and SJR are inventors in a 174 patent application filed by Mayo Clinic relating to the virus described in this report 175 (WO/2018/212842). SJR is a founder and equity holder of Vyriad. The other author has nothing 176 to declare. Data and materials availability: All data needed to evaluate the conclusion of this 177 paper are present in either the main text or the supplementary materials. 
bioRxiv preprint doi: https://doi.org/10.1101/2020.10.22.351189; this version posted October 23, 2020. The copyright holder for this preprint (which was not certified by peer review) is the author/funder. All rights reserved. No reuse allowed without permission.

\section{Supplementary Materials:}

179 Materials and Methods

180 Figures S1-S17 


\section{References}

182 1. M. J. Mina et al., Measles virus infection diminishes preexisting antibodies that offer protection from other pathogens. Science 366, 599-606 (2019).

184 2. P. A. Rota et al., Measles. Nat Rev Dis Primers 2, 16049 (2016).

3. W. H. Organization, Measles fact sheet; https://www.who.int/news-room/factsheets/detail/measles. (2019).

4. V. H. Leonard, G. Hodge, J. Reyes-Del Valle, M. B. McChesney, R. Cattaneo, Measles virus selectively blind to signaling lymphocytic activation molecule (SLAM; CD150) is attenuated and induces strong adaptive immune responses in rhesus monkeys. $J$ Virol 84, 3413-3420 (2010).

5. V. H. Leonard et al., Measles virus blind to its epithelial cell receptor remains virulent in rhesus monkeys but cannot cross the airway epithelium and is not shed. $J$ Clin Invest 118, 2448-2458 (2008).

6. M. J. Birrer, S. A. Udem, S. Nathenson, B. R. Bloom, Antigenic variants of measles virus. Nature 293, 67-69 (1981).

7. S. J. Schrag, P. A. Rota, W. Bellini, Spontaneous mutation rate of measles virus: direct estimation based on mutations conferring monoclonal antibody resistance. J. Virol. 73, 51-54 (1999).

8. B. O. Fulton et al., Mutational Analysis of Measles Virus Suggests Constraints on Antigenic Variation of the Glycoproteins. Cell Rep 11, 1331-1338 (2015).

9. M. Tahara et al., Measles Virus Hemagglutinin Protein Epitopes: The Basis of Antigenic Stability. Viruses 8, (2016).

10. T. Hashiguchi et al., Crystal structure of measles virus hemagglutinin provides insight into effective vaccines. Proc. Natl. Acad. Sci. U S A 104, 19535-19540 (2007).

11. K. P. Greenwood, R. Hafiz, R. S. Ware, S. B. Lambert, A systematic review of human-tohuman transmission of measles vaccine virus. Vaccine 34, 2531-2536 (2016).

12. J. J. Schnorr et al., Measles virus-induced down-regulation of CD46 is associated with enhanced sensitivity to complement-mediated lysis of infected cells. Eur J Immunol 25, 976-984 (1995).

13. M. Takeda et al., Measles viruses possessing the polymerase protein genes of the Edmonston vaccine strain exhibit attenuated gene expression and growth in cultured cells and SLAM knock-in mice. J Virol 82, 11979-11984 (2008).

14. B. Bankamp, M. Takeda, Y. Zhang, W. Xu, P. A. Rota, Genetic characterization of measles vaccine strains. J Infect Dis 204 Suppl 1, S533-548 (2011).

15. M. A. Munoz-Alia, C. P. Muller, S. J. Russell, Hemagglutinin-specific neutralization of subacute sclerosing panencephalitis viruses. PLoS One 13, e0192245 (2018).

16. P. J. Lech et al., Epitope dampening monotypic measles virus hemagglutinin glycoprotein results in resistance to cocktail of monoclonal antibodies. PLoS One 8, e52306 (2013).

17. M. A. Munoz-Alia, C. P. Muller, S. J. Russell, Antigenic Drift Defines a New D4 Subgenotype of Measles Virus. J Virol 91, (2017). 
18. M. A. Muñoz-Alia et al., Measles Virus Hemagglutinin epitopes immunogenic in natural infection and vaccination are targeted by broad or genotype-specific neutralizing monoclonal antibodies. Virus Res 236, 30-43 (2017).

19. Y. P. Liu, S. P. Russell, C. Ayala-Breton, S. J. Russell, K. W. Peng, Ablation of nectin4 binding compromises CD46 usage by a hybrid vesicular stomatitis virus/measles virus. J Virol 88, 2195-2204 (2014).

20. M. Mateo, C. K. Navaratnarajah, S. Syed, R. Cattaneo, The Measles Virus Hemagglutinin beta-Propeller Head beta4-beta5 Hydrophobic Groove Governs Functional Interactions with Nectin- 4 and CD46 but Not Those with the Signaling Lymphocytic Activation Molecule. J Virol 87, 9208-9216 (2013).

234

21. X. Zhang, L. J. Rennick, W. P. Duprex, B. K. Rima, Determination of spontaneous mutation frequencies in measles virus under nonselective conditions. J Virol 87, 26862692 (2013).

22. M. O. Altman, D. Angeletti, J. W. Yewdell, Antibody Immunodominance: The Key to Understanding Influenza Virus Antigenic Drift. Viral Immunol 31, 142-149 (2018). 


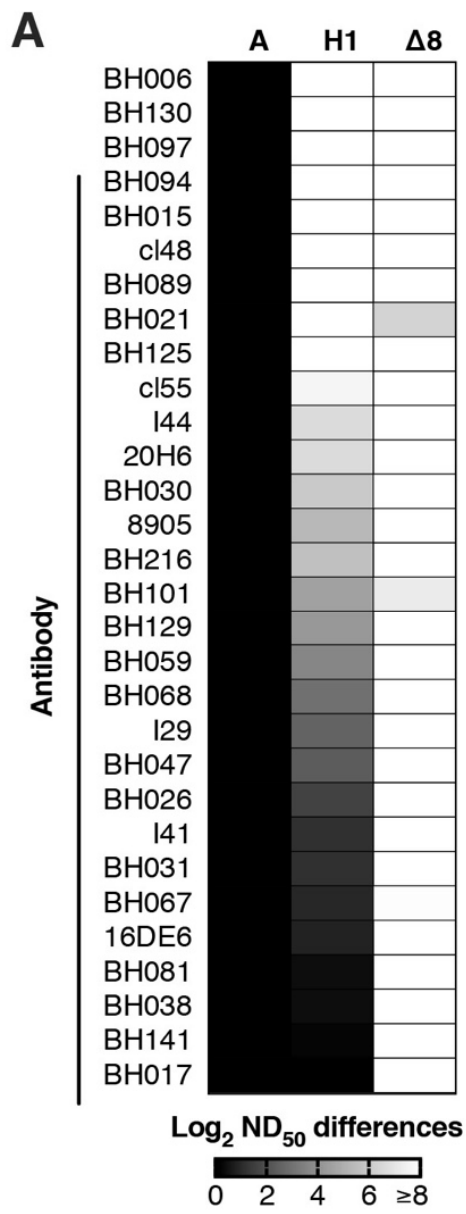

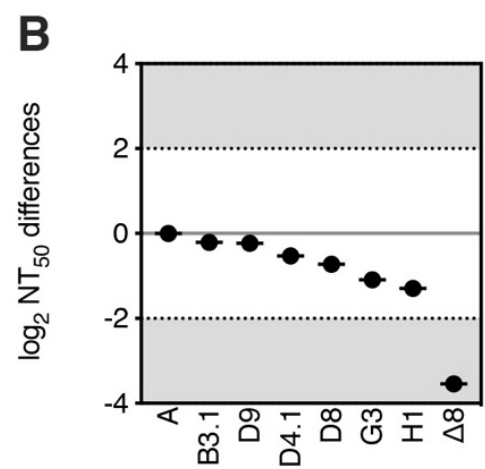

MeV-H encoded into MeV

C

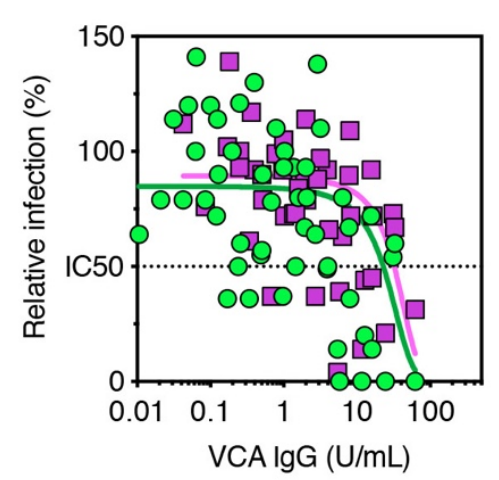

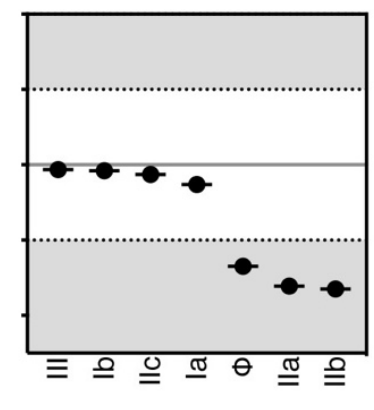

$\mathrm{MeV}-\mathrm{H} \Delta 7$ encoded into $\mathrm{MeV}$

Human sera F depleted

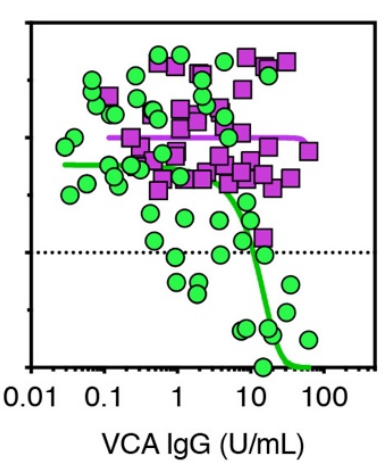

$\left.\begin{array}{l}\because A \\ \square- \\ \square 8\end{array}\right] p=0.0002$

Figure 1. Rational Design of $\mathrm{MeV}-\mathrm{H} \Delta 8$. (A) Neutralization sensitivity of viruses encoding $\mathrm{MeV}-\mathrm{H} \mathrm{A}, \mathrm{H} 1$, and $\Delta 8$ against a panel of $30 \mathrm{mAbs}$. Boxes are shaded according to $\log _{2} \mathrm{ND}_{50}$ reduction. (B, left panel) Neutralization sensitivity of viruses encoding genotype-specific $\mathrm{MeV}-\mathrm{H}$ (left panel) or (B, right panel) $\mathrm{MeV}-\mathrm{H} \Delta 7$ mutants (indicated is the antigenic site remaining intact) against mouse sera post-MeV $\mathrm{A}$ infection. A difference $\geq 2 \log _{2}$ (grey-shaded region) is considered antigenically significant. (C) Neutralization sensitivity of $\mathrm{MeV}$ A or $\Delta 8$ against MeVimmune human sera after depletion of MeV-F-specific antibodies (Fig. S5). Epstein-Barr virus viral capsid antigen (VCA) IgG levels were used to accounting for dilution factors since depletion conditions should not affect their levels. 
A

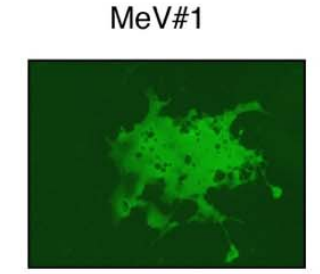

$\mathrm{MeV}$ envelope glycoproteins
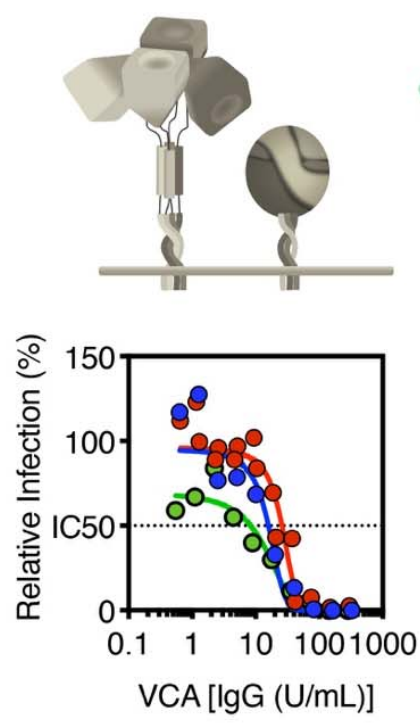

MeV\#2

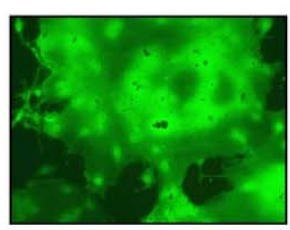

CDV envelope glycoproteins
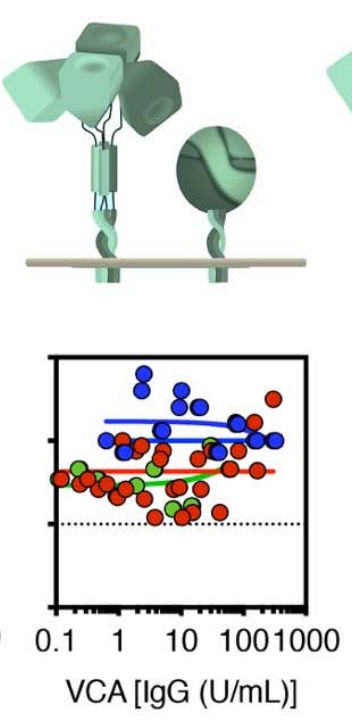

Non absorbed
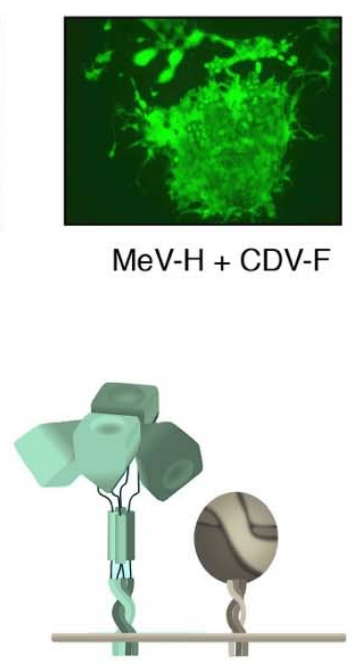

$\mathrm{MeV}-\mathrm{H}+\mathrm{CDV}-\mathrm{F}$

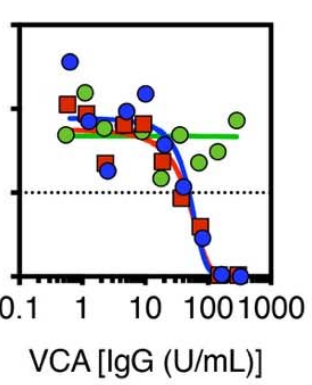

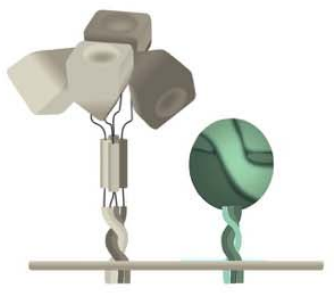

$\mathrm{MeV \# 4}$

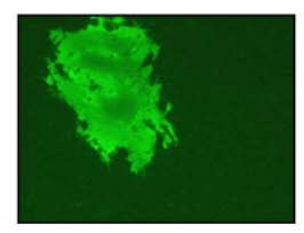

$\mathrm{CDV}-\mathrm{H}+\mathrm{MeV}-\mathrm{F}$

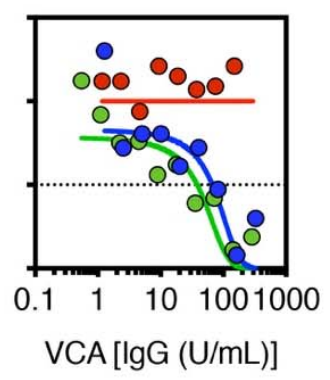

$\mathrm{F}$ absorbed $\mathrm{O} \mathrm{H}$ absorbed

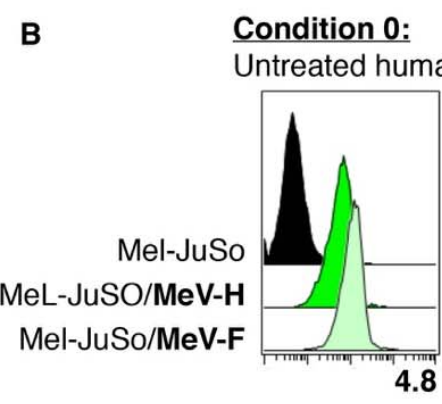

Condition 0:

Untreated human serum

Geometric mean

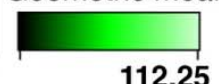

Condition 1:

Mock depletion

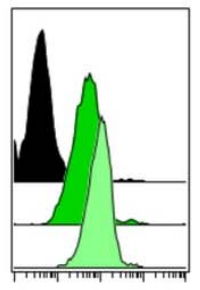

Condition 2:

$\mathrm{MeV}-\mathrm{H}$ depletion

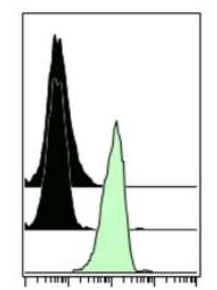

Condition 3:

MeV-F depleted

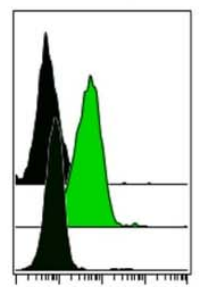

Fig. 2. Role of MeV-H and MeV-F in Virus Neutralization. (A) Virus neutralization assay of envelope-exchange viruses. Isogenic recombinant $\mathrm{MeV}$ encoding $\mathrm{MeV}$ envelope glycoproteins (virus 1), CDV (virus 2), or chimeric viruses (virus 3 and 4) were used to test neutralization sensitivity against pooled human $\mathrm{AB}$ sera depleted of antibodies against MeV-H or MeV-F. Representative syncytia are shown. (B) MeV glycoprotein specificity of pooled human $\mathrm{AB}$ sera. Conditions and IgG specific levels are described and determined as in Figure S5. The remaining $\mathrm{MeV}$ coat-specific antibodies were tested again to confirm successful depletion. Data are shown as histogram plots. 
A

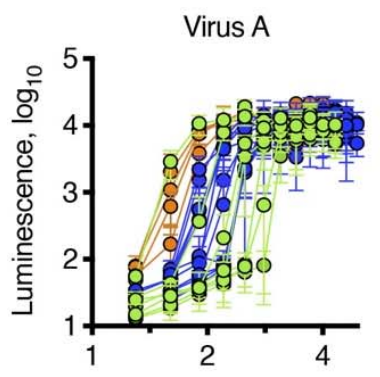

Log10 (reverse of dilution)

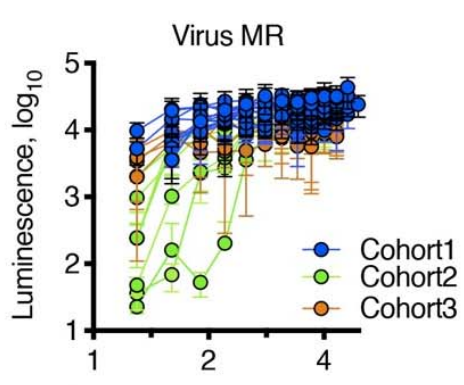

Log10 (reverse of dilution)
B

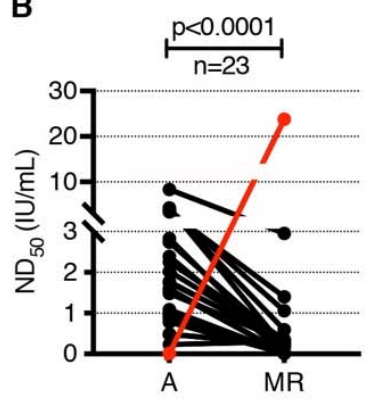

C

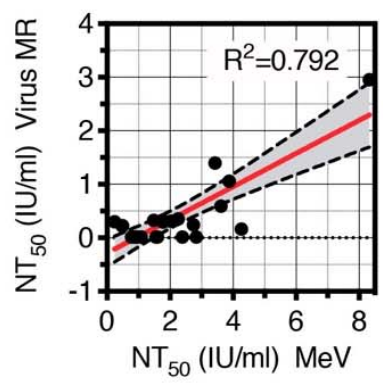

253 Fig. 3. Neutralization activity of human serum samples. (A) Individual neutralization curves.

254 Samples belonging to different cohorts are colored-code. (B) $\mathrm{ND}_{50}$ values of $\mathrm{MeV}$-immune

255 human sera against the MeV A and MR. Each line represents an individual sample $(\mathrm{N}=23)$. The

256 red line shows ferret serum anti-CDV, used as a control for neutralization. Statistical significance

257 was inferred by a two-tailed paired t-test. (C) Correlation between $\mathrm{ND}_{50}$ for the vaccine virus and

258 the MR virus. $\mathrm{P}<0.001$, for both Pearson and Spearman correlation test. The red curve line

259 represents the linear regression line, with dotted lines indicating the 95\% CI for the regression

260 analysis. 
A
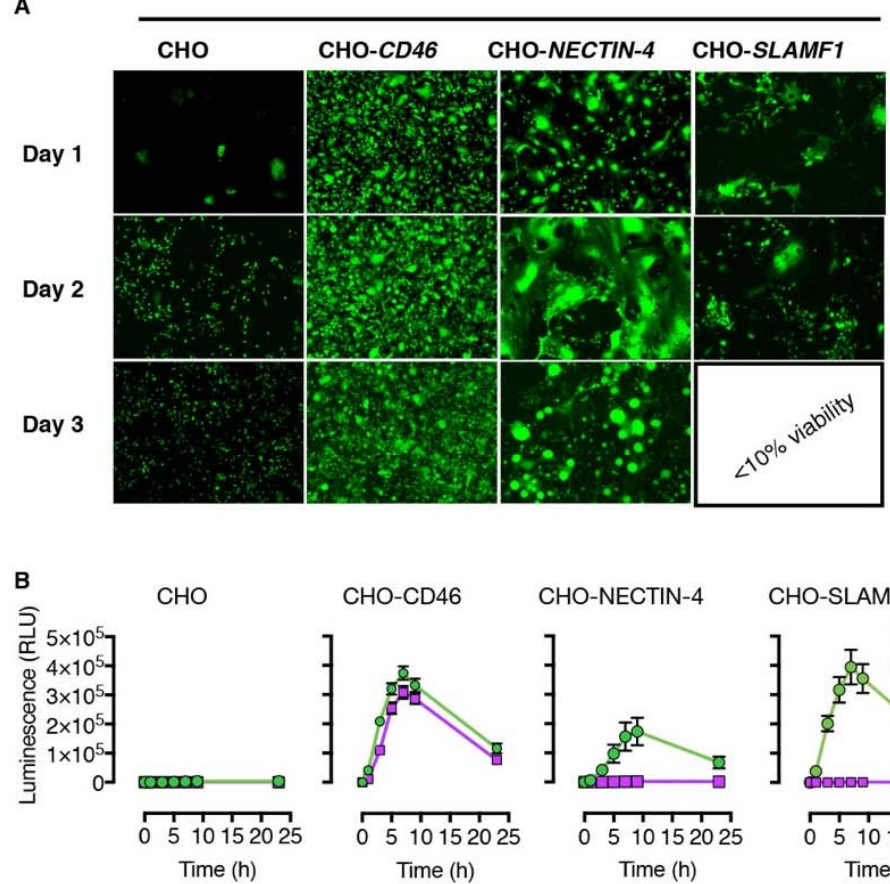

$\mathrm{CHO}-\mathrm{CD} 46$

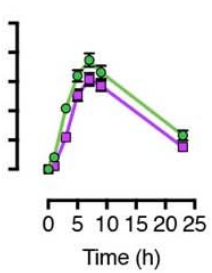

CHO-NECTIN-4

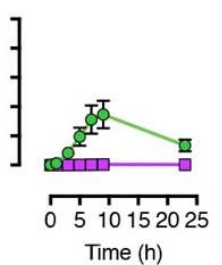

CHO-SLAMF1

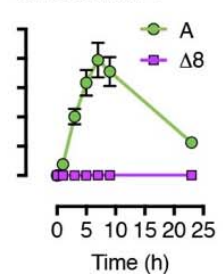

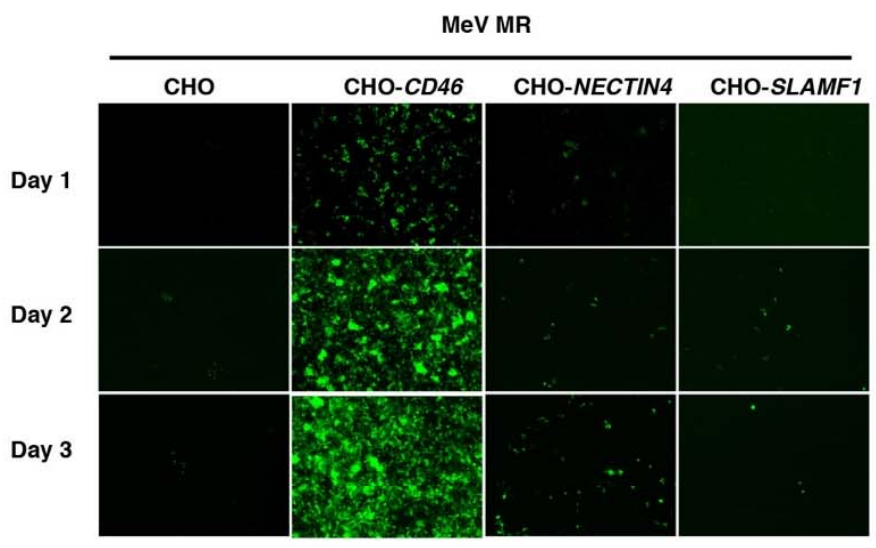

c

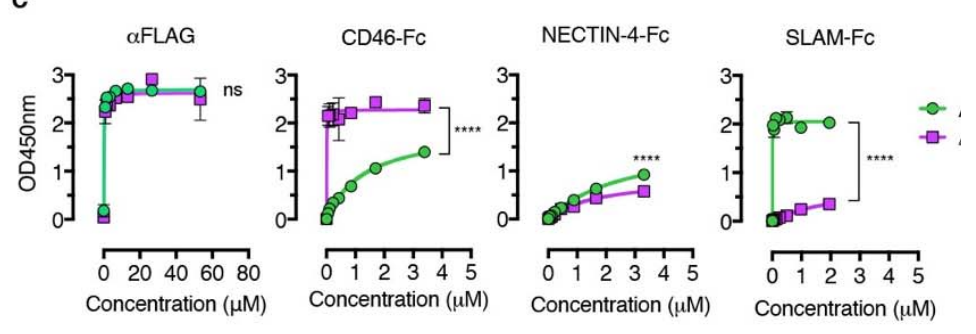

261 Fig 3. Receptor Usage. (A) $\mathrm{CHO}$ cells expressing different $\mathrm{MeV}$ receptors were infected at MOI

262 of 1 . Images were obtained 3 days after infection. Magnification $\times 40$. (B) Kinetics fusion assay

263 after coexpression of MeV-F with either MeV-H A or $\Delta 8$. Mean \pm SD. (C) Binding of MeV

264 receptors-Fc to MeV-H protein, monitored by OD. The FLAG epitope present in both MeV-H

265 was used as a coating control. Data are presented as mean \pm and were fitted to a 1-site mode of 266 total binding $\left(\mathrm{R}^{2} \geq 0.99\right)$. Statistical significance was determined using the Holm-Sidak multiple

267 comparison test: ns, no significant; $* * * * P<.001$. 